Original Research

\title{
The Long-Term Impact of Mild Traumatic Brain Injuries on Multiple Functional Outcomes and Epigenetics: A Pilot Study with College Students
}

\author{
Hyunhwa Lee ${ }^{1, *} \odot$, Sungchul Lee ${ }^{2}$, Ipuna Black ${ }^{3}$, Laura Salado ${ }^{4}$, Jonica Estrada ${ }^{5}$, and Katrina Isla ${ }^{6}$ \\ 1 University of Nevada, Las Vegas, School of Nursing 1; hyunhwa.lee@unlv.edu \\ 2 University of Wisconsin, Whitewater, Computer Science Department 2; lees@uww.edu \\ 3 Nevada State College, School of Nursing 3; ipuna.black@nsc.edu \\ 4 Safe School Professional, Faiss Middle School 4; Saladlc@nv.ccsd.net \\ 5 University of Nevada, Las Vegas, School of Life Sciences 5; estraj1@unlv.nevada.edu \\ 6 University of Nevada, Las Vegas, School of Nursing 6; katrinakeiko@gmail.com \\ * Correspondence: hyunhwa.lee@unlv.edu; Tel.: +1 702-895-3492
}

\section{Featured Application: N/A}

\begin{abstract}
People who suffer a mild traumatic brain injury (mTBI) have heterogeneous symptoms and disease trajectories, which make it difficult to precisely diagnose and assess complications longterm. Insufficient information is available regarding how to precisely diagnose and assess mTBI. This study identified and compared deficits in cognitive, psychosocial, visual functions, and balance performance between college students with and without histories of mTBI. Global DNA methylation ratio (5-mC\%) in blood was also compared as a peripheral epigenetic marker. Twentyfive volunteers participated in this pilot study, including $11 \mathrm{mTBI}$ cases (27.3\% females; mean age of 28.7 years, $\mathrm{SD}=5.92)$ and 14 healthy controls (64.3\% females; mean age of $22.0, \mathrm{SD}=4.13)$. All the participants were assessed for cognitive (by NIH toolbox-executive function, memory, and processing speed), psychological (by PROMIS - depression, anxiety, and sleep disturbances), visual function (by King-Devick and binocular accommodative tests), postural balance performance (by a force plate), and blood 5-mC\% (global methylation) levels. Students with mTBI reported significantly poorer episodic memory, severe anxiety, and more sleep disturbance problems. They also had higher blood 5-mC\% level (all $p^{\prime} s<.05$ ). No significant differences were found in visual function and postural balance. These findings validate changes in cognitive, psychosocial, and global DNA methylation long after mTBI.
\end{abstract}

Keywords: mild traumatic brain injury; mTBI; concussion; cognitive; sensorimotor; visual; postural balance; methylation; 5-mC\%; blood

\section{Introduction}

Mild traumatic brain injury (mTBI), or concussion, is the most common type of traumatic brain injury (TBI) and defined as a traumatically induced physiological disruption of brain function [1], following an injury to the brain. Of nearly 2.8 million TBI cases in the US [2], mild TBI accounts for over $75-80 \%$ cases [3,4]. mTBI is conventionally diagnosed based on the following three criteria: (a) loss of consciousness shorter than $30 \mathrm{~min}$; (b) post-traumatic amnesia shorter than 24 hours; and (c) when the injury is scored 13 to 15 on the Glasgow Coma Scale (GCS) (moderate TBI by 9-12 and severe TBI by 3-8) [1,5]. Although the injury is classified as 'mild', mTBI can cause multifaceted and persistent functional impairments. Such persistent and chronic, often untreated, impairments can severely disturb the quality of life of affected individuals [6]. While most post-mTBI functional deficits may recover in 3 to 6 months [7], up to $40 \%$ of affected individuals [8-10] suffer from 
persistent functional impairments and $25 \%$ cannot return to work 1 year post-injury [9], which causes $\$ 76.5$ billion in economic impact [11]. The increasing incidence and growing health impact of mTBI may result from the lack of or insufficient information regarding how to accurately diagnose and assess it [12].

Heterogeneous symptom development and chronic disease progress following the injury further challenge appropriate screening and treating mTBI. Mood changes [13,14], cognitive performance (in up to $40 \%$ of the affected) [15-17], sensorimotor deficits [18]-such as vision problems $[19,20]$ and impaired balance (in 20 to $80 \%$ of the affected) $[7,21]$ are the most common persistent post-injury functional impairments. Additionally, biomarker changes in neuro-inflammatory (both pro- and antiinflammatory) markers are seen [22]. Changes in global DNA methylation levels are associated with neuro-inflammation and aging [23]. Altered global methylation may indicate the beginning of neurodegeneration by indexing cellular age [24,25]. Specifically, signs and symptoms of neurodegeneration, such as short-term memory loss and balance problems, are associated with changes in methylated regions based on methylome sequencing data of Alzheimer's disease [26], dementia, and Parkinson's disease [27]. Exploring peripheral DNA methylation may contribute to better understanding of the epigenetic regulation of both physical and psychological stress adaptations, which could further inform effective assessments and treatments for mTBI.

For moderate to severe TBIs, standard neuroimaging, including MRI scans, can be used for accurately diagnosing by detecting the gross damage in brain cells [28], however, mTBIs lack common observable brain lesions in MRI scans, and some neurobiological impairments following a mTBI may be undetected without proper instrumentation [29]. The GCS and post-traumatic amnesia are used to aid in diagnosing different severity TBIs, but for evaluating mTBIs neither neuroimaging nor neurobehavioral techniques shows sufficient sensitivity and specificity [30]. The current mTBI assessment, mostly limited to clinical interviews and questionnaires, does not ensure appropriate injury identification and post-injury treatment and is not capable to detect early signs and symptoms of persistent impairments following mTBI. Therefore, such insensitive clinical approaches can lead to elevated risk of both repetitive head traumas and potential long-term brain damage, possibly exacerbating the effects of aging on the brain - premature aging [31] or chronic encephalopathy [32]. The insufficient information in screening post-mTBI impairments lead to misdiagnosis or faulty management for mTBI [12], which may cause major disability or death [33] in the long-term.

Another challenge with treatment is individuals with mTBI may not recognize or refuse to admit post-mTBI symptoms after injury [34]. In addition, some individuals avoid necessary medical screening after mTBI and are reluctant to seek rehabilitation services [35,36]. Moreover, young adults, such as college students, including student athletes and student veterans, typically consider the mTBI diagnosis a stigma and/or a hindrance to their return to play or duty and therefore, avoid screening $[37,38]$. College students with untreated mTBI face the unique pressures of academic success coupled with the demands of reintegration to the civilian life, juggling young families and jobs, and/or rigorous training schedules. Navigating any of these issues with a brain injury, diagnosed or undiagnosed, can be profoundly challenging [39]. Evidence strongly demonstrates the significance of accurate and timely recognition and management for $\mathrm{mTBI}$ in college student population.

Therefore, we proposed a multimodal and systemic approach to post-mTBI neurobiological deficits for college students, including student veterans. The purpose of this pilot study was to identify functional deficits in cognitive, psychosocial, visual, and balance performance for mTBI. Global DNA methylation ratio (5-mC\%) in blood was also compared between individuals with and without a history of mTBI. Analyzing objective functional measures and peripheral epigenetic markers can provide a better understanding of the neurogenerative disease progress following $\mathrm{mTBI}$ and help predict persistent post-injury impairments.

\section{Materials and Methods}


All subjects submitted their informed consent for inclusion before they participated in the study. The study was conducted in accordance with the Declaration of Helsinki, and the protocol was approved by the Biomedical Institutional Review Board Committee at the University of Nevada, Las Vegas (protocol \#1048342). This study had a cross-sectional descriptive design to compare different functional levels and blood $5-\mathrm{mC} \%$ between voluntary college student participants with and without a self-reported history of mTBI. Both males and females aged 18 years or older were recruited. Specific inclusion criteria for mTBI cases were an exposure to a close head (no skull fracture) trauma that resulted in loss of consciousness less than 30 minutes and post-traumatic amnesia less than 24 hours. Those who were medically diagnosed with any other neuro-psychiatric diseases (e.g., multiple sclerosis, ADHD, cerebral palsy) or on non-prescription drugs and alcohol use 24 hours before testing were excluded. This study was advertised by flyers on the UNLV campus as well as via email blasts advertisement to the members of the Military and Veteran Services Center from the director at the center. After an initial phone interview all subjects meeting the inclusion criteria participated in a series of recorded procedures aimed to establish their levels of oculomotor efficacy, cognitive and psychological functions, balance control, and 5-mC\% via blood sample. All procedures took 2.5 hours. Each subject was compensated \$25 cash for his or her time and effort in completing the data collection. Tools and measures used in this study follow.

First, the Rivermead post-concussion symptom questionnaire (RPQ) [40] and the Neurobehavioral symptom inventory (NSI) [41] were used to evaluate overall post-mTBI symptoms. The RPQ contains 16 items on premorbid functioning by means of written self-report or in-person or telephone interview. The RPQ has a high test-retest reliability ( $r$, ranged: 0.87-0.91) [40] and has been used most often in assessing mTBI-related symptoms at all levels of severity-in individuals with mild, moderate, and severe TBI. The NSI has 22 items to cover a comprehensive range of the symptom domains (physical, cognitive, affective, and sensory) [41,42] and has good-to-excellent reliabilities across domains ( $r$, ranged: 0.77-0.93). Both RPQ and NSI have been widely used with various clinical samples with mTBI or concussion, including active military service members and veterans $[41,43,44]$.

The NIH toolbox [45] with computer generated cognitive tasks was used to measure cognitive function. The six following modules from the NIH toolbox were used to evaluated short-term memory, recognition of patterns, and spatial recognition (http://www.healthmeasures.net/exploremeasurement-systems/nih-toolbox): Dimension Change Card Sort Test (DCCS) for executive function, Flanker Inhibitory Control and Attention Test for executive function and attention, Picture Sequence Memory Test for episodic memory, List Sorting Working Memory Test for working memory, Picture Vocabulary Test for vocabulary knowledge, and Pattern Comparison Processing Speed Test for processing speed. Two additional scores were also included: Fluid Cognition Composite Score based on DCCS, Flanker, Picture Sequence, List Sorting, and Processing modules; and Early Childhood Composite Score based on DCCS, Flanker, Picture Sequence, and Picture Vocabulary. All modules were developed and validated by the NIH with state-of-the-science methodology. The NIH toolbox has shown excellent reliability and validity with various populations with good-to-excellent reliabilities ( $r$, ranged: 0.78-0.99) [45].

The Patient Reported Outcomes Measurement Information System (PROMIS) [46] was used to measure the current psychological functions (i.e., depression, anxiety, and sleep disturbances in past 7 days). The PROMIS is a highly reliable, precise measure of patient-reported health status for physical, mental, and social well-being. Three domain measures from PROMIS-depression, anxiety, and sleep disturbances - were used. Each domain measure has undergone rigorous qualitative and psychometric evaluation and refinement through numerous clinical studies, providing efficient, precise, valid, and responsive indicators of a person's health status.

As a standing balance measure, a force platform (FP4060-05-PT) was used to acquire characteristics of bipedal standing balance test. Following the customized method and procedure described in Degani et al. (2017) and Santos et al. (2017) [47,48], we asked the subject to stand still on the force plate with barefoot for 120 seconds with crossing their upper limbs against their chest and focusing their vision on a static point at eye level. The task was performed while the subject stood 
vertically on the top of a force platform while the coordinates of the body's Center of Pressure (CoP) were recorded. The force plate provided data of horizontal and vertical components of the ground reaction force $(\mathrm{Fx}, \mathrm{Fy}, \mathrm{Fz})$ and the moments of force around the frontal, sagittal and vertical axes $(\mathrm{Mx}$, $\mathrm{My}, \mathrm{Mz}$ ). These data were used to compute postural sway based on instantaneous CoP location during the standing task in both anterior-posterior and medio-lateral directions (CoPAP and CoPML, respectively). Adapting Degani et al. (2017) and Santos et al. (2017) [47,48], the following variables of interest were extracted from $\mathrm{CoP}$ coordinates and used in analysis: the area of $\mathrm{CoP}$ path (Area), the peak-to-peak amplitude of the CoP displacement in each direction (AmplitudeAP and AmplitudemL), the mean velocity of the CoP displacement in each direction $\left(M V_{A P}\right.$ and $\left.M V_{M L}\right)$, the frequency at which $80 \%$ of the CoP spectral power for each direction ( $F 80_{A P}$ and $F 80_{M L}$ ), the sample entropy estimates of the CoP displacement in each direction (SEnt $A_{A P}$ and $S E n t_{M L}$ ), and the cross-sample entropy (CrossSEnt) representing the degree of asynchrony or dissimilarity between CoPAP and CoPML signals in time.

For examining visual function, a trained optometric assistant screened each subject's visual function using the two following approaches: (1) King-Devick Oculomotor Testing - Involved testing the eye's ability to "track" or move accurately and efficiently from one point to the next [49]. The test has three sections, which include numbers with guides, numbers without guides, and numbers without guides and with irregular spacing. The subject was timed while reading the numbers left to right, and errors were monitored until the subject completed reading aloud each slide. Initially derived by an optometrist, the test is becoming an increasing popular interdisciplinary test among professionals working with individuals with mTBI or concussion [49,50]. (2) Binocular Accommodative Facility with +2.00/-2.00 Flipper Lens - Testing the ability of the eye's ability to accommodate (or, "focus" and "make clear") when simulating near and far distances using a special set of lenses. The visual system's ability to dynamically focus at different distances (i.e., with close proximity while reading or at the distance when looking toward a street sign) is a visual skill, which may be impacted in mTBI. Inefficiencies may be demonstrated by perceiving double vision upon adjusting focus through the lens, inability to clear a lens (either minus which simulates near focus, or the plus lens which simulates distance focus), or an increased length of time to clear either or both sides.

Blood 5-mC\% measure. Following the procedures approved by the UNLV Institutional Biosafety Committee, a trained phlebotomist drew a total of $12 \mathrm{ml}$ of blood in three $4 \mathrm{~mL}$ EDTA tubes via venipuncture from each participant in the designated lab on campus. The collected whole blood samples were stored in the freezer at $-70^{\circ} \mathrm{C}$ in the Applied Biomedical Research Lab (ABRL) until DNA purification. Using QIAamp Blood DNA extraction kit (Qiagen, Germantown, MD), cell-free DNA samples were isolated from collected whole blood following the manufacturer's manuals at the ABRL. Purified cell-free genomic DNA samples were stored in the freezer at $-70^{\circ} \mathrm{C}$ in the ABRL until methylation assay. MethylFlash ${ }^{\mathrm{TM}}$ Methylated DNA Quantification Kit (Epigentek, Farmingdale, NY) was used to quantify global DNA methylation by specifically measuring levels of 5-methylcytosine (5-mc) in an enzyme-linked immunosorbent assay-based microplate-based format in the ABRL. All samples will be read at the absorbance of $450 \mathrm{~nm}$ (Epoch, Biotek U.S., Winooski, VT) for global DNA methylation levels (absolute percentages).

Data Analysis. All data were statistically analyzed using the Statistical Package for the Social Sciences (SPSS) version 22.0. The balance data from the force plate were first processed off-line by customized MATLAB software (MATLAB, Mathworks Inc., Natick, MA), before being transferred into the SPSS software. Descriptive statistics for all demographic and clinical variables were calculated. Any variability in the distribution of age, sex, and other demographic variables were examined by independent $\mathrm{t}$-tests. Functional deficits in mTBI cases, compared to healthy controls', independent $\mathrm{t}$ tests were used. Analysis of covariance (ANCOVA) were used to control for potential covariates such as age and sex. All statistic tests were carried following the statistic rigor imposed by each of the tests and levels of significance were kept at $5 \%(\alpha=0.05)$.

\section{Results}


Twenty-five volunteer college students participated $(M=25.0$ years of age; $S D=5.96$ years $)$ in this study. The demographic characteristics of the participants are given in Table 1. mTBI cases consisted of 11 individuals (27.3\% females; mean age of 28.7 years, SD = 5.92) with a self-reported history of single or multiple injuries (63.6\% multiple; $2.3 \pm 1.25$ injuries). The traumatic occurrences include sports-related activities and military operations. The average time elapsed since the last injury was $7.1(\mathrm{SD}=6.77)$ years. Controls consisted of 14 individuals $(64.3 \%$ females; $M=22.0$ years of age; $S D=4.13$ years). Only age has a statistical difference between TBI and controls $(p=.007)$ among all demographic variables.

Table 1. Demographic differences $(n(\%)$ or $M \pm S D$ ) between mTBI and healthy control college student participants.

\begin{tabular}{|c|c|c|c|c|c|}
\hline Characteristic & Overall $(n=25)$ & $\operatorname{mTBI}(n=11)$ & Control $(n=14)$ & $x^{2}$ or $t$ & $p$ \\
\hline Age & $24.96 \pm 5.96$ & $28.73 \pm 5.92$ & $22.00 \pm 4.13$ & 3.348 & 0.003 \\
\hline \multicolumn{6}{|l|}{ Gender } \\
\hline Males & $12(50)$ & $8(72.7)$ & $5(35.7)$ & 3.381 & 0.066 \\
\hline Females & $12(50)$ & $3(27.3)$ & $9(64.3)$ & & \\
\hline Weight (kg) & $70.85 \pm 16.77$ & $75.67 \pm 14.97$ & $67.07 \pm 17.66$ & 1.129 & 0.210 \\
\hline Height (cm) & $169.06 \pm 11.81$ & $173.87 \pm 10.62$ & $165.28 \pm 11.64$ & 1.903 & 0.070 \\
\hline \multicolumn{6}{|l|}{ Ethnicity/Race } \\
\hline Hispanic & $4(16.0)$ & $2(18.2)$ & $2(14.3)$ & 0.263 & 0.992 \\
\hline White & $14(56.0)$ & $6(54.5)$ & $8(57.1)$ & & \\
\hline Black & $2(8.0)$ & $1(9.1)$ & $1(7.1)$ & & \\
\hline Asian & $3(12.0)$ & $1(9.1)$ & $2(14.3)$ & & \\
\hline Other & $2(8.0)$ & $1(9.1)$ & $1(7.1)$ & & \\
\hline \multicolumn{6}{|l|}{ Handedness } \\
\hline Right & $22(88.0)$ & $9(81.8)$ & $13(92.9)$ & 0.711 & 0.399 \\
\hline Left & $3(12.0)$ & $2(18.2)$ & $1(7.1)$ & & \\
\hline \multicolumn{6}{|l|}{ Education } \\
\hline In college & $22(88.0)$ & $10(90.9)$ & $12(85.7)$ & 0.834 & 0.659 \\
\hline In graduate school & $3(12.0)$ & $1(9.1)$ & $2(14.3)$ & & \\
\hline \multicolumn{6}{|l|}{ Marital Status } \\
\hline Single & $21(84.0)$ & $9(81.8)$ & $12(92.6)$ & 1.376 & 0.503 \\
\hline Married & $3(12.0)$ & $2(18.2)$ & $1(7.7)$ & & \\
\hline \multicolumn{6}{|l|}{ Employment Status } \\
\hline Yes & $16(64.0)$ & $5(45.5)$ & $10(71.4)$ & 0.762 & 0.383 \\
\hline No & $9(36.0)$ & $6(54.5)$ & $4(28.6)$ & & \\
\hline \multicolumn{6}{|l|}{ Income } \\
\hline Low $(\leq \$ 50 \mathrm{~K})$ & $13(52.0)$ & $6(54.2)$ & $7(58.3)$ & 1.703 & 0.427 \\
\hline $\begin{array}{l}\text { Middle }(\$ 50 \mathrm{~K}< \\
\leq \$ 100 \mathrm{~K})\end{array}$ & $6(24.0)$ & $2(18.2)$ & $4(33.3)$ & & \\
\hline High $(\$ 100 \mathrm{~K}<)$ & $4(16.0)$ & $3(27.3)$ & $1(8.3)$ & & \\
\hline
\end{tabular}

Table 2 depicts functional differences between mTBI and healthy control groups. mTBI cases reported significantly poorer age-corrected episodic memory (mTBI, $99.00 \pm 17.19$; controls, $115.57 \pm$ 
15.64; $p=.022$ ), severer anxiety (mTBI, $56.55 \pm 9.77$; controls, $49.00 \pm 5.09 ; p=.022$ ) and sleep disturbance problems (mTBI, $56.34 \pm 11.82$; controls, $45.89 \pm 9.17 ; p=.023$ ), along with higher total scores in both RPQ and NSI. When comparing each post-mTBI symptom item in both RPQ and NSI, 18 out of 22 NSI items (e.g., dizziness with body balance, visual, cognitive, and psychological dysfunctions etc.), and 14 out of 16 RPQ items (e.g., headache, dizziness, cognitive, psychological, and visual dysfunctions, etc.) showed significantly higher item scores in mTBI, compared to control group (all $p^{\prime} \mathrm{s}<0.05$ ). When controlling for age by one-way ANCOVA, these was no significant effect of having mTBI history on sleep disturbance problems. However, the differences in anxiety $[F(1,23)$ $=6.331, p=.020]$, RPQ $[F(1,24)=23.103, p=.000]$ and NSI $[F(1,23)=11.280, p=.003]$ scores were still significant. There were no statistically significant differences found in visual and postural balance performances between the two groups.

Table 2. Functional differences $(M \pm S D)$ between $\mathrm{mTBI}$ and healthy control college student participants.

\begin{tabular}{|c|c|c|c|c|c|}
\hline Functional variables & Overall $(n=25)$ & mTBI $(n=11)$ & Control $(n=14)$ & $t$ & $p$ \\
\hline \multicolumn{6}{|c|}{ Cognitive Function by NIH Toolbox (age corrected): } \\
\hline $\begin{array}{l}\text { Dimension Change Card } \\
\text { Sort Test (DCCS) }\end{array}$ & $98.17 \pm 18.83$ & $97.20 \pm 15.85$ & $98.86 \pm 21.26$ & -0.208 & 0.837 \\
\hline $\begin{array}{l}\text { Flanker Inhibitory } \\
\text { Control and Attention } \\
\text { Test }\end{array}$ & $83.67 \pm 14.64$ & $82.10 \pm 15.99$ & $84.79 \pm 14.11$ & -0.435 & 0.668 \\
\hline $\begin{array}{l}\text { Picture Sequence } \\
\text { Memory Test }\end{array}$ & $108.67 \pm 17.99$ & $99.00 \pm 17.19$ & $115.57 \pm 15.64$ & -2.457 & 0.022 \\
\hline $\begin{array}{l}\text { List Sorting Working } \\
\text { Memory Test }\end{array}$ & $103.92 \pm 12.25$ & $103.40 \pm 11.94$ & $104.29 \pm 12.90$ & -0.171 & 0.866 \\
\hline Picture Vocabulary Test & $110.08 \pm 10.97$ & $113.60 \pm 7.59$ & $107.57 \pm 12.51$ & 1.3511 & 0.190 \\
\hline $\begin{array}{l}\text { Pattern Comparison } \\
\text { Processing Speed Test }\end{array}$ & $102.75 \pm 22.52$ & $107.70 \pm 20.30$ & $99.21 \pm 24.08$ & 0.906 & 0.375 \\
\hline $\begin{array}{l}\text { Fluid Cognition } \\
\text { Composite Score }\end{array}$ & $99.08 \pm 15.73$ & $96.70 \pm 18.89$ & $100.79 \pm 13.54$ & -0.619 & 0.542 \\
\hline $\begin{array}{l}\text { Early Childhood } \\
\text { Composite Score }\end{array}$ & $99.96 \pm 14.25$ & $96.80 \pm 15.33$ & $102.21 \pm 13.53$ & -0.915 & 0.370 \\
\hline \multicolumn{6}{|c|}{ Psychological Function by PROMIS: } \\
\hline Depression & $50.73 \pm 7.26$ & $54.04 \pm 7.57$ & $48.37 \pm 6.26$ & 2.005 & 0.057 \\
\hline Anxiety & $52.15 \pm 8.15$ & $56.55 \pm 9.77$ & $49.00 \pm 5.09$ & 2.472 & 0.022 \\
\hline Sleep Disturbance & $50.24 \pm 11.4$ & $56.34 \pm 11.82$ & $45.89 \pm 9.17$ & 2.637 & 0.015 \\
\hline \multicolumn{6}{|l|}{$\underline{\text { Visual Function by }}$} \\
\hline \multicolumn{6}{|l|}{ King-Devick Test: } \\
\hline Number of misses & $0.04 \pm 0.20$ & $0.00 \pm 0.00$ & $0.07 \pm 0.27$ & -0.840 & 0.410 \\
\hline Total time (sec.) & $50.73 \pm 22.11$ & $57.66 \pm 31.68$ & $45.29 \pm 7.64$ & 1.417 & 0.170 \\
\hline \multicolumn{6}{|l|}{ Accommodation Test: } \\
\hline Number of tests per min & $10.96 \pm 3.55$ & $10.59 \pm 3.00$ & $11.25 \pm 4.01$ & -0.453 & 0.654 \\
\hline Body Balance Performance & a force platform: & & & & \\
\hline
\end{tabular}




\begin{tabular}{lccccc} 
Area $\left(\mathrm{cm}^{2}\right)$ & $1.96 \pm 1.73$ & $2.23 \pm 2.16$ & $1.76 \pm 1.39$ & 0.652 & 0.521 \\
AmplitudeAP $_{\left(\mathrm{cm}^{2}\right)}$ & $1.71 \pm 1.12$ & $2.09 \pm 1.41$ & $1.44 \pm 0.81$ & 1.428 & 0.167 \\
AmplitudeML $_{\left(\mathrm{cm}^{2}\right)}$ & $2.62 \pm 1.09$ & $2.32 \pm 0.77$ & $2.84 \pm 1.24$ & -1.149 & 0.263 \\
MeanVelocity $_{A P}\left(\mathrm{~cm} \mathrm{~s}^{-1}\right)$ & $3.10 \pm 1.34$ & $3.49 \pm 1.55$ & $2.82 \pm 1.17$ & 1.222 & 0.068 \\
MeanVelocityML $_{\left(\mathrm{cm} \mathrm{s}^{-1}\right)}$ & $5.31 \pm 1.72$ & $5.16 \pm 2.10$ & $5.41 \pm 1.47$ & -0.339 & 0.738 \\
F80AP $(\mathrm{Hz})$ & $0.45 \pm 0.43$ & $0.52 \pm 0.64$ & $0.40 \pm 0.19$ & 0.709 & 0.486 \\
F80 & $0.26 \pm 0.10$ & $0.27 \pm 0.08$ & $0.25 \pm 0.11$ & 0.437 & 0.666 \\
SEnt & $0.56 \pm 0.19$ & $0.53 \pm 0.14$ & $0.59 \pm 0.22$ & -0.724 & 0.477 \\
SEnt & $0.52 \pm 0.13$ & $0.54 \pm 0.12$ & $0.51 \pm 0.14$ & 0.575 & 0.571 \\
CrossSEnt & $1.86 \pm 1.24$ & $1.56 \pm 1.06$ & $2.07 \pm 1.35$ & -0.993 & 0.332 \\
Overall post-mTBI symptoms by & & & & & \\
RPQ Total score & $11.71 \pm 14.28$ & $24.40 \pm 13.83$ & $2.64 \pm 3.95$ & 5.617 & $\mathbf{0 . 0 0 0}$ \\
NSI Total score & $13.57 \pm 14.43$ & $24.80 \pm 15.02$ & $4.92 \pm 5.16$ & 4.469 & $\mathbf{0 . 0 0 0}$ \\
\hline
\end{tabular}

We also examined the differences by the number of injuries by further dividing the mTBI group - single vs. multiple injuries. The multiple injury group reported significantly older age (32.43 $\pm 2.51)$ than the single injury group $(22.25 \pm 4.03)[t(9)=-5.239, p=.001]$. Although differences were found in Area and MeanVelocityap from the force plate balance test between the two injury groups, those effects disappeared when controlling for age by one-way ANCOVA. Only the age-corrected Pattern Comparison Processing Speed Test score for processing speed was significantly lower in the multiple injury group $(n=7 ; 96.33 \pm 18.23)$ than in the single injury group $(n=4 ; 124.75 \pm 6.02)[t(8)=$ $2.960, p=.018]$.

Peripheral epigenetic marker comparison finding showed significantly higher blood global methylation level $(5-\mathrm{mC} \%)$ in mTBI cases $(4.42 \pm 0.43)$ than in controls $(3.93 \pm 0.42)(p=.030)$, as shown in Figure 1. This difference still existed after controlling for age by one-way ANCOVA $[F(1,15)=$ $6.276, p=.026]$.

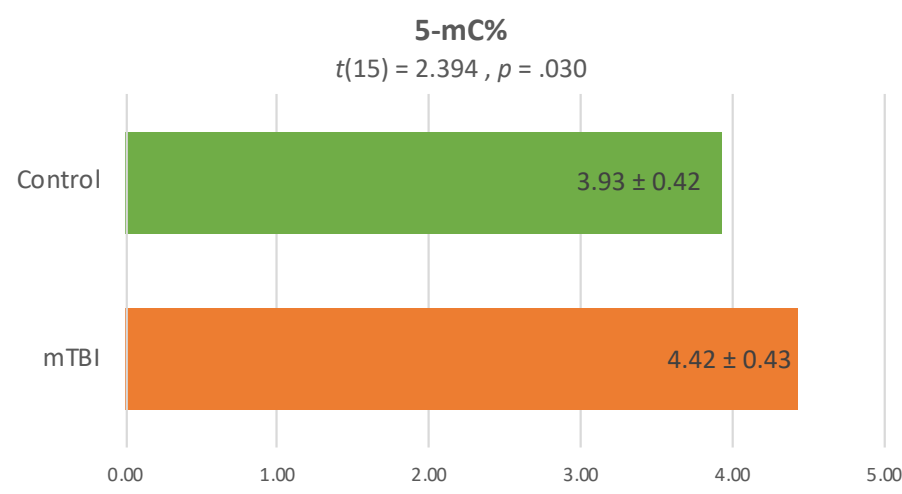

Figure 1. Comparison of peripheral blood global methylation level (5-mC\%) between mTBI and healthy control college student participants.

\section{Discussion}

In the present study, we used a comprehensive range of rapid, non-invasive, and objective cognitive, neuropsychological, and sensorimotor functional measures, along with blood 5-mC $\%$ level, to compare and identify functional deficits and underlying biological factors in symptom expression and complications resulting from mTBI among a group of college students. Even several years after the injury (7.1 years, on average, elapsed since the last injury), the 11 students with mTBI histories 
(mean age of 28.7 years, $\mathrm{SD}=5.92$ ) showed significantly poorer episodic memory, severer anxiety, and more sleep disturbance problems than 14 healthy control students $(M=22.0$ years of age; $S D=$ 4.13). Higher peripheral global DNA methylation ratio $(5-\mathrm{mC} \%)$ was also found in $\mathrm{mTBI}$ group, compared with control group. The mTBI history effect became insignificant on sleep problems only when controlling for age.

Our results of post-mTBI cognitive deficits highlight that college students who reported mTBI histories showed poorer episodic memory, than their counterpart controls. We employed 6 different modules of the NIH toolbox to examine the college students' executive function, attention, episodic and working memories, vocabulary knowledge, and processing speed. Those with mTBI histories showed significantly lower scores on the Picture Sequence Memory Test for episodic memory only, both age-corrected and -uncorrected, but not the other test modules nor any of cognitive composite scores. When comparing multiple to single injury within the mTBI cases, we found that the multiple injury group showed the significantly lower score of the age-corrected Pattern Comparison Processing Speed Test for processing speed than the single injury group. Episodic memory, which is involved in storage of distinctive events or experiences encoded in a time-specific manner [45], is one of the key indices to determine premature aging in several neurodegenerative disorders, such as Alzheimer's disease [24]. Our finding on poorer episodic memory post-mTBI confirm the previous findings from MRI and CT studies with clinical TBI patients [31,51]. As shown in a recent study comparing over 80 young and older individuals with mTBI [52], declines in episodic memory may serve as an early indicator of post-mTBI premature aging among young adults like college students. Lower processing speed, which is one of the other main indices of brain aging along with declined executive function, was confirmed only when comparing the multiple injury group to the single injury group of our study. In a national study with over 200 professional fighters, lower processing speed and lower brain volumes were associated with multiple or repetitive head trauma [53], which supports our findings among college students although the sample size in the present study was small. Both episodic memory and processing speed are fundamental elements of human cognitive function, and deficits in either or both may assist more effective and sensitive screening for disrupted brain function following mTBI.

We also found that, post mTBI, college students suffered from psychosocial complications long after the injury, particularly anxiety and sleep disturbances based on PROMIS measures. There was the effect of age on the post-mTBI sleep disturbance problems in this study, but different formats of sleep problems (e.g., unable to fall asleep, daytime drowsiness, sleep too much or little) have been found in larger samples of young individuals with mTBI [54,55]. In a recent qualitative study, a quarter of the participants, part-time or full-time undergraduate and graduate students enrolled at a large state university who have suffered one or more mTBI, reported sleep cycle disturbances [56]. Although no statistical significance was found in difference of depression symptom scores between mTBI and control groups, our findings support the current evidence that mTBI is associated with mood changes, irritability, anxiety, and poor anger management [57-59]. More specifically, college students have reported an increase in crying over situations they may not have prior to the mTBI and less likely to take risks because of how they will be perceived [56]. Persons with mTBI can experience challenges with using feedback and interpreting social cues, resulting in social inappropriate behavior [60]. These behaviors and personality shifts contribute to loss of friendships and close relationships. In one study, about half of the college participants with a history of mTBI reported difficulty with maintaining relationships [61]. Without connections, these college students lack a sense of belonging, which can increase the risk for developing mood disorders, and both increase suicide risk [62].

We could not identify any functional deficits of visual or postural balance performances in mTBI group, using King-Devick and accommodation tests or force-platform postural balance test, although those individuals rated relevant post-mTBI symptom items in both RPQ and NSI checklists significantly higher. When mTBI causes changes in the cortex and brainstem's reticular formation [63], different parts of sensory input may have altered interaction with each other, which further 
disrupts the functioning of the postural control system and causes postural instability [64]. Specific symptoms include impairments of visual, vestibular, or somatosensory orientation [65]. Although both King-Devick test and force-platform postural balance test are most widely used to assess sensorimotor functional levels, they may not be sensitive enough to detect functional changes after mTBI, compared to moderate or severe TBI. Unsupervised approach with a wider detectable range for functional variability, such as wearable sensor devices [66], and a more systemic eye tracking method $[67,68]$ may be recommended for more effective and precise assessment of postural balance and visual functions in mTBI.

For overall post-mTBI symptom profiles, we used the NSI and the RPQ that rate each of different potential post-injury symptoms, including physical, cognitive, affective, and sensory functional changes or deficits. Both are self-report tools, but the difference is the reflection time frame. The NSI asked each participant to compare potential post-mTBI symptoms over the last 2 weeks with functional status before the initial injury, whereas the RPQ asked the similar thing over the last 24 hours. A cut-off of 24 for the total NSI score has been identified to provide clinically meaningful assessment results [41], The multiple injury subgroup in our pilot study scored higher than 24 on the NSI $(27.86 \pm 17.74)$, whereas the single injury scored lower than that $(21.50 \pm 5.75)$, although there was no statistical significance. For the RPQ, a cut-off of 16 was reported to show high sensitivity and specificity for persistent post-injury symptoms [69], but both multiple and single injury subgroups in this pilot study scored higher than 16. Other study reported that the data from NSI and RPQ could be combined or converted with each other in chronic TBI sample [70], but our findings cautiously suggest the possibility that the longer reflection period in the NSI may better differentiate the chronic impact of multiple injuries vs. single injury. Indeed, repetitive head injuries have been associated higher risks for severer post-injury symptoms, including cognitive deficits as also shown in our data, and further developing neurological disorders, such as chronic traumatic encephalopathy [53,71]

In our knowledge, our study is one of the first to examine peripheral global DNA methylation in association with mTBI, which could contribute to better understanding of underlying biological mechanisms of post-injury symptoms. We found that individuals with mTBI had significantly higher levels of blood global methylation ratio $(5-\mathrm{mC} \%)$ than healthy controls and the significance remains when controlling for age. Because of changes in the blood-brain barrier permeability and the entire gliovascular unit after mTBI, blood biomarkers may be developed into clinical monitoring tools to detect brain damage [10]. Even when repetitive or multiple head injuries over time are involved, which might not be accurately assessed, epigenetic changes, such as in global DNA methylation, can be the best biomarker to detect pathologic process after mTBI because epigenetic mechanisms have long-lasting effects within mature neurons without altering the DNA code [72]. Usually located in a gene promoter, DNA methylation typically acts to repress gene transcription [73]. DNA methylation is essential for normal development and is associated with a number of key processes including aging and carcinogenesis (i.e., global hypomethylation) [74,75]. Because our participants were cancer-free, their increased global methylation ratio could be a unique biomarker related to mTBI and/or postinjury functional declines.

The present study has limitations although the main findings support the changes in cognitive, psychosocial, and global DNA methylation after mTBI. The sample size is small in this pilot study. Based on the current findings, a larger sample should be examined in a future study to confirm the effectiveness of our comprehensive functions screening and peripheral epigenetic test. Past mTBI events were assessed based on the college student participants' self-report. Although we used a structured interview questionnaire including the GCS and post-traumatic amnesia information, many participants were either unaware of those clinical characteristics/measures or incompetent with their recalling memory on such information. Yet, this is not an unusual situation with individuals who suffered from mTBI, as the majority appears to avoid necessary post-injury screening in a clinical setting [35,36], especially among college student athletes and student veterans who consider the mTBI diagnosis a stigma and/or a hindrance to their return to play or duty and therefore, avoid screening $[37,38]$. In addition, the King-Devick and accommodation visual tests and force-platform postural 
stability test could not identify any differences between mTBI cases and controls. As discussed before, those measures may not be sensitive enough to detect mTBI-specific functional changes. In future studies, with increased sample sizes, unsupervised and more systemic approach to detect a wider range of functional variability, such as wearable sensor devices [66] and an eye tracking method $[67,68]$, is recommended.

Regardless, our main findings revealed several functional deficits long after mTBI, which could contribute to early detection of mTBI symptoms for ensuring early intervention and rehabilitation. In particular, college student athletes and veterans with untreated mTBI face the unique pressures of academic success, coupled with the demands of reintegration to the civilian life, juggling young families and jobs, and/or rigorous training schedules. Navigating any of these issues with a brain injury, diagnosed or undiagnosed, can be profoundly challenging, but very important [39]. There are no available diagnostic tests to assess recovery and no proven treatments to reduce functional damages from mTBI. Our findings based on the comprehensive and systemic neuropsychological measures along with peripheral epigenetic markers provide strong evidence to addresses underlying neuropathophysiology and peripheral epigenetic factors in symptom expression and complications resulting from $\mathrm{mTBI}$, which can be used for predicting and monitoring functional progress and for future clinical interventions.

\section{Conclusions}

In the present study, we could identify decreased episodic memory, anxiety, more sleep problems, and higher $5-\mathrm{mC} \%$ in blood in college students with $\mathrm{mTBI}$, which were still significant, except for sleep problems, when controlling for age. Those with multiple injuries further showed lower processing speed than those with single injury. Focused assessment on cognitive and psychosocial functions along with peripheral epigenetic markers may contribute to the minimization of the related morbidity and mortality risks, and the improvement of quality of life in affected individuals.

Author Contributions: Conceptualization, H.L.; methodology, H.L.; software, H.L. and S.L.; validation, H.L., S.L.; formal analysis, H.L and S.L.; investigation, H.L. and S.L.; resources, L.S., S.L., J.E., and K.I.; data curation, H.L., L.S., S.L., J.E., and K.I.; writing-original draft preparation, H.L.; writing-review and editing, I.B., S.L., L.S., J.E., and K.I.; visualization, H.L.; supervision, H.L.; project administration, H.L., L.S., S.L., J.E., and K.I.; funding acquisition, H.L..

Funding: This research was funded by University of Nevada, Las Vegas (UNLV), School of Nursing.

Acknowledgments: This study was supported by the UNLV School of Nursing and the 2017-2020 UNLV Top Tier Doctoral Research Graduate Assistantship. Blood samples were collected via venipuncture at the UNLV Student Wellness Center. The force plate method and data analysis procedures were advised by Dr. Alessander Santos (https://wmich.edu/physicaltherapy/directory/santos).

Conflicts of Interest: The authors declare no conflict of interest. The funders had no role in the design of the study; in the collection, analyses, or interpretation of data; in the writing of the manuscript, or in the decision to publish the results.

\section{References}

1. American Congress of Rehabilitation Medicine Definition of mild traumatic brain injury. J. Head Trauma Rehabil. 1993, 8, 86-87.

2. Taylor, C.A.; Bell, J.M.; Breiding, M.J.; Xu, L. Traumatic brain injury-related emergency department visits, hospitalizations, and deaths - United States, 2007 and 2013. MMWR. Surveill. Summ. 2017, 66, 116.

3. Kay, A.; Teasdale, G. Head Injury in the United Kingdom. World J. Surg. 2001, 25, 1210-1220.

4. National Center for Injury Prevention and Control Report to Congress on mild traumatic brain injury in the 
United States: Steps to prevent a serious public health problem; Atlanta, GA, 2003;

5. Teasdale, G.; Jennett, B. Assessment of coma and impaired consciousness: A practical scale. Lancet 1974, $304,81-84$.

6. Seabury, S.A.; Gaudette, É.; Goldman, D.P.; Markowitz, A.J.; Brooks, J.; McCrea, M.A.; Okonkwo, D.O.; Manley, G.T.; Adeoye, O.; Badjatia, N.; et al. Assessment of follow-up care after emergency department presentation for mild traumatic brain injury and concussion. JAMA Netw. Open 2018, 1, e180210.

7. Katz, D.I.; White, D.K.; Alexander, M.P.; Klein, R.B. Recovery of ambulation after traumatic brain injury. Arch. Phys. Med. Rehabil. 2004, 85, 865-9.

8. McCrea, M.A. Mild traumatic brain injury and postconcussion syndrome: The new evidence base for diagnosis and treatment; Oxford University Press: New York, 2008;

9. Langlois, J.A.; Rutland-Brown, W.; Wald, M.M. The epidemiology and impact of traumatic brain injury. J. Head Trauma Rehabil. 2006, 21, 375-378.

10. Bennett, E.R.; Reuter-Rice, K.; Laskowitz, D.T. Genetic influences in traumatic brain injury. In Translational Research in Traumatic Brain Injury; Laskowitz D, G.G., Ed.; CRC Press/Taylor and Francis Group, 2016 ISBN 9781466584914.

11. Centers for Disease Control and Prevention CDC grand rounds: Reducing severe traumatic brain injury in the United States. Morb. Mortal. Wkly. Rep. 2013, 549-552.

12. Sharp, D.J.; Jenkins, P.O. Concussion is confusing us all. Pract. Neurol. 2015, 15, 172-86.

13. Lee, H.; Lee, S.; Salado, L.; Estrada, J.; Danna-dos-Santos, A.; Isla, K.; Lao, J.; Mohapatra, S.; Bernick, C. Long-term impact of mild traumatic brain injuries on cognitive, psychosocial and balance performance and epigenetics. In Proceedings of the Proceedings of the 2018 Asian American/Pacific Islander Nurses Association Conference: Local to Global--Future Directions for Research on Health Disparities; Durham, North Carolina., 2018.

14. Gill, J.; Lee, H.; Barr, T.; Baxter, T.; Heinzelmann, M.; Rak, H.; Mysliwiec, V. Lower health related quality of life in U.S. military personnel is associated with service-related disorders and inflammation. Psychiatry Res. 2014, 216, 116-122.

15. Luethcke, C.A.; Bryan, C.J.; Morrow, C.E.; Isler, W.C. Comparison of concussive symptoms, cognitive performance, and psychological symptoms between acute blast-versus nonblast-induced mild traumatic brain injury. J. Int. Neuropsychol. Soc. 2011, 17, 36-45.

16. Giza, C.C.; Hovda, D.A. The new neurometabolic cascade of concussion. Neurosurgery 2014, 75, S24-S33.

17. McInnes, K.; Friesen, C.L.; MacKenzie, D.E.; Westwood, D.A.; Boe, S.G. Mild traumatic brain injury (mTBI) and chronic cognitive impairment: A scoping review. PLoS One 2017, 12, e0174847.

18. Tremblay, S.; de Beaumont, L.; Lassonde, M.; Théoret, H. Evidence for the specificity of intracortical inhibitory dysfunction in asymptomatic concussed athletes. J. Neurotrauma 2011, 28, 493-502.

19. Ciuffreda, K.J.; Kapoor, N.; Rutner, D.; Suchoff, I.B.; Han, M.E.; Craig, S. Occurrence of oculomotor dysfunctions in acquired brain injury: A retrospective analysis. Optom. - J. Am. Optom. Assoc. 2007, 78, 155-161.

20. Thiagarajan, P.; Ciuffreda, K.J.; Ludlam, D.P. Vergence dysfunction in mild traumatic brain injury (mTBI): A review. Ophthalmic Physiol. Opt. 2011, 31, 456-468.

21. Valovich McLeod, T.C.; Hale, T.D. Vestibular and balance issues following sport-related concussion. Brain Inj. 2015, 29, 175-184.

22. Lee, H.; Merchant-Borna, K.; Wang, D.; Bogner, V.; van Griensven, M.; Gill, J.; Bazarian, J.J. Genome- 
wide changes in peripheral gene expression following sports-related concussion. J. Neurotrauma 2016, 33, 1576-85.

23. Jung, M.; Pfeifer, G.P. Aging and DNA methylation. BMC Biol. 2015, 13, 7.

24. Barter, J.D.; Foster, T.C. Aging in the brain: New roles of epigenetics in cognitive decline. Neurosci. 2018, $24,516-525$.

25. Chouliaras, L.; Pishva, E.; Haapakoski, R.; Zsoldos, E.; Mahmood, A.; Filippini, N.; Burrage, J.; Mill, J.; Kivimäki, M.; Lunnon, K.; et al. Peripheral DNA methylation, cognitive decline and brain aging: Pilot findings from the Whitehall II imaging study. Epigenomics 2018, 10, 585-595.

26. Villela, D.; Ramalho, R.F.; Silva, A.R.T.; Brentani, H.; Suemoto, C.K.; Pasqualucci, C.A.; Grinberg, L.T.; Krepischi, A.C.V.; Rosenberg, C. Differential DNA methylation of microRNA genes in temporal cortex from Alzheimer's disease individuals. Neural Plast. 2016, 2016.

27. Sanchez-Mut, J. V; Heyn, H.; Vidal, E.; Moran, S.; Sayols, S.; Delgado-Morales, R.; Schultz, M.D.; Ansoleaga, B.; Garcia-Esparcia, P.; Pons-Espinal, M.; et al. Human DNA methylomes of neurodegenerative diseases show common epigenomic patterns. Transl. Psychiatry 2016, 6, e718-e718.

28. McCrea, M.; Meier, T.; Huber, D.; Ptito, A.; Bigler, E.; Debert, C.T.; Manley, G.; Menon, D.; Chen, J.-K.; Wall, R.; et al. Role of advanced neuroimaging, fluid biomarkers and genetic testing in the assessment of sport-related concussion: a systematic review. Br. J. Sports Med. 2017, 51, 919-929.

29. Centers for Disease Control and Prevention Report to Congress on traumatic brain injury in the United States: Epidemiology and rehabilitation; Atlanta, GA, 2015;

30. Berger, R.P. The use of serum biomarkers to predict outcome after traumatic brain injury in adults and children. J. Head Trauma Rehabil. 2006, 21, 315-333.

31. Tremblay, S.; Henry, L.C.; Bedetti, C.; Larson-Dupuis, C.; Gagnon, J.F.; Evans, A.C.; Théoret, H.; Lassonde, M.; De Beaumont, L. Diffuse white matter tract abnormalities in clinically normal ageing retired athletes with a history of sports-related concussions. Brain 2014, 137, 2997-3011.

32. Zetterberg, H.; Blennow, K. Fluid biomarkers for mild traumatic brain injury and related conditions. Nat. Rev. Neurol. 2016, 12.

33. Tator, C.H. Concussions and their consequences: Current diagnosis, management and prevention. CMAJ 2013, 185, 975-979.

34. Centers for Disease Control and Prevention Traumatic brain injury \& concussion: Signs and symptoms Available online: https://www.cdc.gov/traumaticbraininjury/symptoms.html (accessed on Sep 27, 2018).

35. Dams-O'Connor, K.; Cuthbert, J.P.; Whyte, J.; Corrigan, J.D.; Faul, M.; Harrison-Felix, C. Traumatic brain injury among older adults at level I and II trauma centers. J. Neurotrauma 2013, 30, 2001-2013.

36. McQuistion, K.; Zens, T.; Jung, H.S.; Beems, M.; Leverson, G.; Liepert, A.; Scarborough, J.; Agarwal, S. Insurance status and race affect treatment and outcome of traumatic brain injury. J. Surg. Res. 2016, 205, 261-271.

37. Peskind, E.R.; Brody, D.; Cernak, I.; McKee, A.; Ruff, R.L. Military- and sports-related mild traumatic brain injury: Clinical presentation, management, and long-term consequences. J. Clin. Psychiatry 2013, 74, 180-8; quiz 188.

38. Iverson, G.L.; Lange, R.T. Mild traumatic brain injury. In The Little Black Book of Neuropsychology; Springer US: Boston, MA, 2011; pp. 697-719.

39. Glang, A.; Ettel, D.; Todis, B.; Gordon, W.A.; Oswald, J.M.; Vaughn, S.L.; Connors, S.H.; Brown, M. Services and supports for students with traumatic brain injury: Survey of state educational agencies. 
Exceptionality 2015, 23, 211-224.

40. King, N.S.; Crawford, S.; Wenden, F.J.; Moss, N.E.; Wade, D.T. The Rivermead Post Concussion Symptoms Questionnaire: A measure of symptoms commonly experienced after head injury and its reliability. J. Neurol. 1995, 242, 587-92.

41. Soble, J.R.; Silva, M.A.; Vanderploeg, R.D.; Curtiss, G.; Belanger, H.G.; Donnell, A.J.; Scott, S.G. Normative data for the neurobehavioral symptom inventory (NSI) and post-concussion symptom profiles among TBI, PTSD, and nonclinical samples. Clin. Neuropsychol. 2014, 28, 614-632.

42. Cicerone, K.D.; Kalmar, K. Persistent postconcussion syndrome: The structure of subjective complaints after mild traumatic brain injury. J. Head Trauma Rehabil. 1995, 10, 1-17.

43. Seabury, S.A.; Gaudette, É.; Goldman, D.P.; Markowitz, A.J.; Brooks, J.; McCrea, M.A.; Okonkwo, D.O.; Manley, G.T.; Adeoye, O.; Badjatia, N.; et al. Assessment of follow-up care after emergency department presentation for mild traumatic brain injury and concussion. JAMA Netw. Open 2018, 1, e180210.

44. Belanger, H.G.; Silva, M.A.; Donnell, A.J.; McKenzie-Hartman, T.; Lamberty, G.J.; Vanderploeg, R.D. Utility of the Neurobehavioral Symptom Inventory as an outcome measure: A VA TBI Model Systems Study. J. Head Trauma Rehabil. 2017, 32, 46-54.

45. Weintraub, S.; Dikmen, S.S.; Heaton, R.K.; Tulsky, D.S.; Zelazo, P.D.; Bauer, P.J.; Carlozzi, N.E.; Slotkin, J.; Blitz, D.; Wallner-Allen, K.; et al. Cognition assessment using the NIH Toolbox. Neurology 2013, 80, S54-64.

46. PROMIS Network PROMIS: Dynamic tools to measure health outcomes from the patient perspective Available online: http://www.nihpromis.org.

47. Degani, A.M.; Santos, M.M.; Leonard, C.T.; Rau, T.F.; Patel, S.A.; Mohapatra, S.; Danna-dos-Santos, A. The effects of mild traumatic brain injury on postural control. Brain Inj. 2017, 31, 49-56.

48. Santos, M. Changes in postural sway behavior across the life span; 2017;

49. Galetta, K.M.; Brandes, L.E.; Maki, K.; Dziemianowicz, M.S.; Laudano, E.; Allen, M.; Lawler, K.; Sennett, B.; Wiebe, D.; Devick, S.; et al. The King-Devick test and sports-related concussion: Study of a rapid visual screening tool in a collegiate cohort. J. Neurol. Sci. 2011, 309, 34-39.

50. Ventura, R.; Balcer, L.; Galetta, S. The Concussion Toolbox: The role of vision in the assessment of concussion. Semin. Neurol. 2015, 35, 599-606.

51. Dikmen, S.; Machamer, J.; Temkin, N. Mild traumatic brain injury: Longitudinal study of cognition, functional status, and post-traumatic symptoms. J. Neurotrauma 2017, 34, 1524-1530.

52. Wammes, J.D.; Good, T.J.; Fernandes, M.A. Autobiographical and episodic memory deficits in mild traumatic brain injury. Brain Cogn. 2017, 111, 112-126.

53. Bernick, C.; Banks, S.J.; Shin, W.; Obuchowski, N.; Butler, S.; Noback, M.; Phillips, M.; Lowe, M.; Jones, S.; Modic, M. Repeated head trauma is associated with smaller thalamic volumes and slower processing speed: The professional fighters' brain health study. Br. J. Sports Med. 2015, 49, 1007-11.

54. McCarthy, M.T.; Kosofsky, B.E. Clinical features and biomarkers of concussion and mild traumatic brain injury in pediatric patients. Ann. N. Y. Acad. Sci. 2015, 1345, 89-98.

55. Sullivan, K.A.; Edmed, S.L.; Allan, A.C.; Karlsson, L.J.E.; Smith, S.S. Characterizing self-reported sleep disturbance after mild traumatic brain injury. J. Neurotrauma 2015, 32, 474-486.

56. Childers, C.; Hux, K. Invisible injuries: The experiences of college students with histories of mild traumatic brain injury; 2016; Vol. 29;.

57. Covassin, T.; Elbin, R.J.; Beidler, E.; LaFevor, M.; Kontos, A.P. A review of psychological issues that may 
be associated with a sport-related concussion in youth and collegiate athletes. Sport. Exerc. Perform. Psychol. 2017, 6, 220-229.

58. Thastum, M.M.; Rask, C.U.; Naess-Schmidt, E.T.; Jensen, J.S.; Frederiksen, O.V.; Tuborgh, A.; Svendsen, S.W.; Nielsen, J.F.; Schröder, A. Design of an early intervention for persistent post-concussion symptoms in adolescents and young adults: A feasibility study. NeuroRehabilitation 2018, 43, 155-167.

59. Hux, K.; Bush, E.; Zickefoose, S.; Holmberg, M.; Henderson, A.; Simanek, G. Exploring the study skills and accommodations used by college student survivors of traumatic brain injury. Brain Inj. 2010, 24, 1326.

60. Carulli, L.A.; Olney, M.F.; Degeneffe, C.E.; Conrad, G. Social engagement among community college students with traumatic brain injury. J. Rehabil. 2018, 84, 29-38.

61. Kennedy, M.R.T.; Krause, M.O.; Turkstra, L.S. An electronic survey about college experiences after traumatic brain injury. NeuroRehabilitation 2008, 23, 511-520.

62. Bryson, C.N.; Cramer, R.J.; Schmidt, A.T. Traumatic brain injury and lifetime suicidality: Applying the interpersonal-psychological theory perspective. Death Stud. 2017, 41, 399-405.

63. Shaw, N.A. The neurophysiology of concussion. Prog. Neurobiol. 2002, 67, 281-344.

64. Guskiewicz, K.M. Balance assessment in the management of sport-related concussion. Clin. Sports Med. 2011, 30, 89-102.

65. Ingersoll, C.D.; Armstrong, C.W. The effects of closed-head injury on postural sway. Med. Sci. Sports Exerc. 1992, 24, 739-43.

66. Lee, H.; Lee, S.; Salado, L.; Estrada, J.; White, J.; Muthukumar, V.; Lee, S.-P.; Mohapatra, S. Proof-ofconcept testing of a real-time mHealth measure to estimate postural control during walking: A potential application for mild traumatic brain injuries. Asian / Pacific Isl. Nurs. J. 2019, 3.

67. U.S. Food and Drug Administration Product classification: Brain injury adjunctive interpretive oculomotor assessment Available online: https://www.accessdata.fda.gov/scripts/cdrh/cfdocs/cfpcd/classification.cfm?id=3820 (accessed on Aug 10, 2019).

68. Voelker, R. Eye-Tracking Test Approved to Help Diagnose Concussion. JAMA 2019, 321, 638.

69. Thompson, C.; Davies, P.; Herrmann, L.; Summers, M.; Potter, S. Approaches to establishing validated cut-off scores on the Rivermead post-concussion symptoms questionnaire (RPQ). In Proceedings of the International Brain Injury Association's Eleventh World Congress on Brain Injury; Taylor \& Francis: The Netherlands, 2016.

70. Vos, L.; Whiteneck, G.G.; Ngan, E.; Leon Novelo, L.; Harik, L.M.; Sherer, M. Comparison of the Neurobehavioral Symptom Inventory and the Rivermead Postconcussion Symptoms Questionnaire. Brain Inj. 2019, 33, 1165-1172.

71. Montenigro, P.H.; Alosco, M.L.; Martin, B.M.; Daneshvar, D.H.; Mez, J.; Chaisson, C.E.; Nowinski, C.J.; Au, R.; McKee, A.C.; Cantu, R.C.; et al. Cumulative head impact exposure predicts later-life depression, apathy, executive dysfunction, and cognitive impairment in former high school and college football players. J. Neurotrauma 2017, 34, 328-340.

72. Tsankova, N.; Renthal, W.; Kumar, A.; Nestler, E.J. Epigenetic regulation in psychiatric disorders. Nat. Rev. Neurosci. 2007, 8, 355-367.

73. Lee, H.; Gill, J.; Barr, T.; Yun, S.; Kim, H. Primer in genetics and genomics, article 2-Advancing nursing research with genomic approaches. Biol. Res. Nurs. 2017, 19, 229-239. 
74. Wen, K.; Miliç, J.; El-Khodor, B.; Dhana, K.; Nano, J.; Pulido, T.; Kraja, B.; Zaciragic, A.; Bramer, W.M.; Troup, J.; et al. The role of DNA methylation and histone modifications in neurodegenerative diseases: A systematic review. PLoS One 2016, 11, e0167201.

75. Bellizzi, D.; D'Aquila, P.; Montesanto, A.; Corsonello, A.; Mari, V.; Mazzei, B.; Lattanzio, F.; Passarino, G. Global DNA methylation in old subjects is correlated with frailty. Age (Omaha). 2012, 34, 169-179. 